\title{
IJIMI
}

International Journal of Machine Intelligence

ISSN: 0975-2927 \& E-ISSN: 0975-9166, Volume 3, Issue 3, 2011, pp-112-115

Available online at http://www.bioinfo.in/contents.php?id=31

\section{STUDY OF STRUCTURAL PATTERNS EXHIBITED BY A BINARY MIXTURE OF SMECTIC AND CHOLESTERYL COMPOUNDS}

\author{
GOVINDAIAH T.N. ${ }^{* 1}$, SREEPAD H.R. ${ }^{1}$ AND NAGAPPA ${ }^{2}$ \\ ${ }^{1}$ Research Centre, Government College (Autonomous), Mandya - 571401, Karnataka State, India \\ 2Department of Studies in Physics, University of Mysore, Manasagangotri, Mysore - 570006, Karnataka, India \\ *Corresponding Author: Email- tngovi.phy@gmail.com
}

Received: September 29, 2011; Accepted: November 03, 2011

\begin{abstract}
The binary mixture of Cholesteryl ethyl corbonate (CEC) and Terephthal-bis-p-n-Tetradecylaniline (TB14A) exhibits a twisted grain boundary (TGB) phase and smectic polymorphic phases. The mixture exhibits TGB-A and TGB-C phases in different concentrations and at different temperatures. Mixtures with higher concentrations of TB14A exhibit I - Ch - TGB-A TGB-C - Sm A - Sm I - Sm F phases sequentially when the specimen is cooled from its isotropic melt. The existence of different TGB phases and polymorphic smectic phases are confirmed by DSC and optical microscopic studies.
\end{abstract}

Key words - Optical textures, Renn and Lubbensky model, Smectic and Cholesteric mixture, TGB phase,

\section{Introduction}

Liquid crystals are widely used in electro-optic display devices such as optical switches, light modulators and image devices [1-3]. The use of liquid crystals in these devices depends on the kinds of mesophases exhibited by the liquid crystals, transition temperature (Tc) and optical anisotropies.

The phenomenon associated with the chiral liquid crystals have shown increasing importance with respect to both their fundamental scientific significance and their applicability to electro-optic and opto-electronic applications. The nature of chiral smectic phases that are exhibited in the mixture when there is a phase transition from cholesteric to smectic phase is clearly investigated. The existence of twisted grain boundary (TGB) phase, near a virtual NAC point in the binary mixture of liquid crystalline compounds [4] has been well investigated by earlier investigators.

The theoretical prediction of the Renn and Lubbensky coined a remarkable development in the field of liquid crystals to understand the effect of chirality on the smectic-A liquid crystal $[5,6]$. The theoretical basis for this prediction was the de Gennes model for the nematic to smectic-A (Sm A) transition, which is analogous to super-conducting transition [7]. According to de Gennes model the twisted grain boundary phase (TGB), which occurred on smectic-A side i.e TGB-A phase, is the analog of the Abrikosov [8] vortex lattice in type II super conductors. At the same time, Goodby et. al., [9, 10] discovered the Sm A* phase in highly chiral homologous series. X-ray studies on non-aligned samples reveal that the $S m A^{*}$ phase exhibits both smectic layering and cholesteric like textures simultaneously. Thus the $\mathrm{Sm} \mathrm{A}$ phase is nothing but a TGB phase. The TGB phase is characterized by the non-vanishing twist and long-range smectic correlations with in this plane perpendicular to the pitch axis. It has formed several remarkable features of the Sm A - Sm A* - Sm C $C^{*}$ multicritical point [11]. Nagappa et. al., [12] have observed TGB-A phase in the mixtures of cholesteric and nematic compounds. Sadashiva [13] and Pramod et. al., [14] have observed a transition from TGB-A - TGB-C* in some pure and mixtures of the chiral liquid crystals.

In the present investigation we have studied the binary mixture of smectic and cholesteric compounds. The pure smectic compound exhibits I- Sm C-Sm I-Sm G-Sm Fcrystal phases. At very low concentration of the cholesteric, the mixture exhibits a transition Cho-TGB-A and TGB-A-TGB-C at higher temperature. The DSC and optical texture studies lend support to the above results. All the structural patterns exhibited by the mixture have been identified.

\section{Experimental}

In the present investigation we have used the binary mixtures of smectic and cholesteric liquid crystals, viz., Terephthal-bis-p-n-Tetradecylaniline ( $\left.\mathrm{T}_{\mathrm{B} 14 \mathrm{~A}}\right)$ and Cholesteryl ethyl corbonate (CEC). These chemicals are obtained from M/s Eastman organic chemicals, USA with a given purity of $98 \%$ minimum. In case of $\mathrm{T}_{\mathrm{B} 14 \mathrm{~A}}$ the isotropic-liquid crystalline phase transition temperature is


isotropic-liquid crystalline phase transition temperature is $103^{\circ} \mathrm{C}$, which are measured using polarizing microscope in conjunction with specially constructed hot stage.

For the optical texture studies the sample was sandwiched between the slide and cover slip and melted by keeping it in a conventional hot-stage, the temperature of which can be controlled. The textures were observed using the polarizing microscope by 
varying the temperatures of hot-stage, the thickness of the samples were usually of the order of 25-50 microns.

The isotropic-liquid crystalline phase transition temperatures of $\mathrm{T}_{\mathrm{B} 14 \mathrm{~A}}$ and $\mathrm{CEC}$ are compared with the values obtained from DSC thermograms. The DSC thermograms are obtained from DSC II Instrument facility available at Raman Research Institute, Bangalore, India. The binary mixture of twenty different concentrations of $\mathrm{T}_{\mathrm{B} 14 \mathrm{~A}}$ and $\mathrm{CEC}$ by weight percentage were prepared and mixed very well in the molten state

\section{Results and Discussion \\ Phase diagram}

The sequence of phases displayed at different temperatures in mixtures with $70 \%$ and $50 \%$ of $T_{B 14 A}$ in CEC respectively are given below;

$\mathrm{I} \rightarrow 156.7^{\circ} \mathrm{C}, \mathrm{Cho} \rightarrow 155^{\circ} \mathrm{C}$, TGB-A $\rightarrow 154.1^{\circ} \mathrm{C}$, TGB$\mathrm{C} \rightarrow 152.5^{\circ} \mathrm{C}, \mathrm{Sm} \mathrm{A} \rightarrow 116.4^{\circ} \mathrm{C}, \mathrm{Sm} \mathrm{I} \rightarrow 87.9^{\circ} \mathrm{C}, \mathrm{Sm}$ $\mathrm{F} \rightarrow 68.7^{\circ} \mathrm{C}$ and

$\mathrm{I} \rightarrow 130^{\circ} \mathrm{C}, \mathrm{Sm} \mathrm{A} \rightarrow 96.2^{\circ} \mathrm{C}, \mathrm{Sm} \mathrm{F} \rightarrow 78.2^{\circ} \mathrm{C}$.

\section{Optical Texture studies}

The pure cholesteryl ethyl carbonate (CEC) compound exhibits a planar texture, when there is phase transition from isotropic to liquid crystalline state. The planar texture is characterized by the specular reflection arising from the helicoidal structure of cholesteric phase. This has a spatial periodicity in the order of the wavelength of light.

\section{Recognition of structural patterns}

Various optical textures shown by the mixture at different concentrations are shown in Figs. 1(a to g).

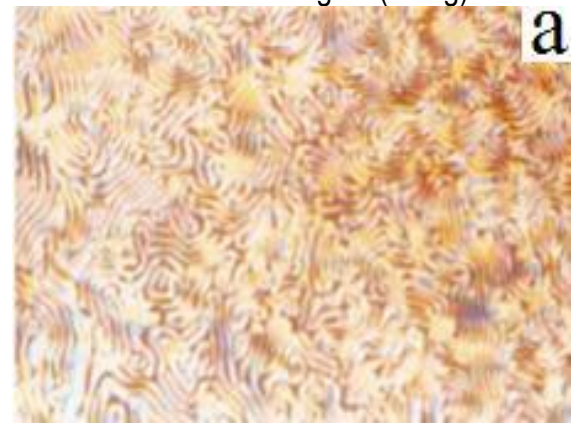

Fig. 1(a) Microphotograph showing Finger print pattern of cholesteric phase (180X).

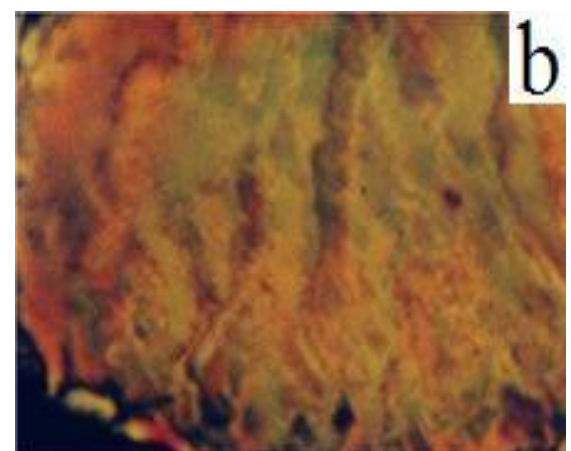

Fig. 1(b) Microphotograph showing Hexagonal grid pattern of TGB-A phase (360X).

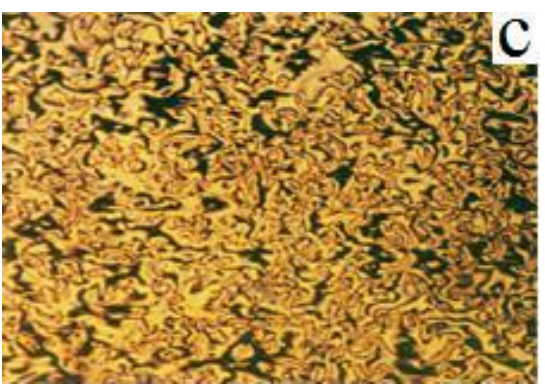

Fig. 1(C) Microphotograph showing Schlieren textures of TGB-C phase (320X)

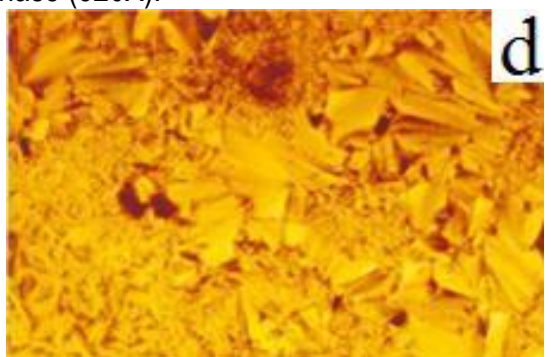

Fig. 1(d) Microphotograph showing Focal conic fan shaped texture of smectic-A phase (320X).

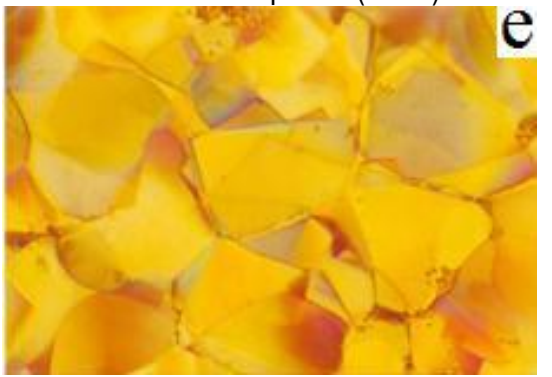

Fig. 1(e) Microphotograph showing Smectic-l phase (220X).

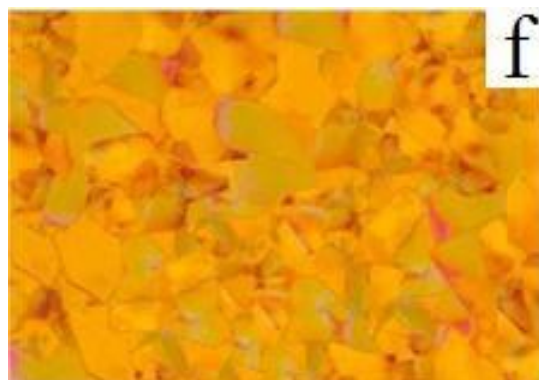

Fig. 1(f) Microphotograph showing Smectic-F phase (220X).

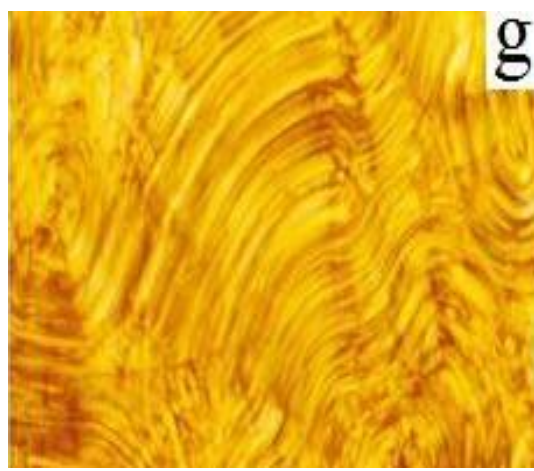

Fig. 1(g) Microphotograph showing Chiral smectic-G phase (220X). 
When the molten sample of mixture of $70 \%$ of $T_{B 14 A}$ in $\mathrm{CEC}$ is cooled from its isotropic-liquid crystalline phase transition temperature $\left(156.7{ }^{\circ} \mathrm{C}\right)$, a genesis of nucleation starts in the form of minute bubbles and immediately these bubbles grow radially and form a finger print pattern, which is the characteristic of cholesteric phase and the texture so obtained is shown in Figure 1(a).

With further decreasing the temperature of the sample, the cholesteric phase changes over to smectic phase passing through an intermediate phase. The transition to the intermediate phase is assigned by the appearance of streak like texture in the homeotropic region, arranged in hexagonal form, which is the characteristic of TGB-A phase and the same is shown in Figure 1(b).

The hexagonal grid patterned twisted grain boundary (TGB-A) phase changes over to the schlieren textures at temperature $152.5^{\circ} \mathrm{C}$ which is the characteristic of TGB$\mathrm{C}$ phase and it appears as shown in Figure 1(c).

On further cooling, the schlieren texture of TGB phase changes over to the focal conic fan shaped texture of smectic-A phase at temperature $116.4^{\circ} \mathrm{C}$ as shown in Figure $1(d)$. Ultimately the $S m A$ phase undergoes a polymorphic smectic phase transition sequentially in the order Sm I-Sm F-Sm G-Crystal phase at lower temperature. The molecules in Sm $\mathrm{G}$ phase are packed with in the layers having their long axes tilted with respect to normal to the layer planes [15].

Whenever TGB-C phase changes over to smectic-A phase, latter this phase is followed by broken focal conic schlieren and schlieren mosaic texture, which are characterized by $\mathrm{Sm} \mathrm{I}$, and Sm F phases are shown in Figures $1(\mathrm{e})$ and $1(\mathrm{f})$ respectively. Here the smectic-I phase is a tilted biaxial phase [16], the pseudo hexagonal molecular packing identifies the molecular structure in the smectic-I phase and this phase is hexatic in nature [17]. In Sm F phase the molecules are packed in layers, with their long axes tilted with respect to the layer planes [18].

The mixture with higher concentrations of $T_{B 14 A}$ and $C E C$ exhibits a broken, banded focal conic fan texture of the chiral smectic-G phase as shown in Figure $1(\mathrm{~g})$. If the constituent molecules of the material, which exhibits a smectic-G phase are of a chiral nature, then the phase itself may also be weakly optically active; it is then termed a chiral smectic-G phase. The structural studies have been carried out at that time on chiral smectic-G phases and it was originally simply presumed that the structure of the phase is similar to that of chiral smectic$\mathrm{C}$, smectic-I and smectic- $\mathrm{F}$ phases. In this case the molecules would be hexagonally close packed in layers within each of which the tilts must be in the same direction. In the layer above and below the tilt direction will however be turned through a small angle.

Thus on passing from layer to layer, the tilt direction will turn slowly either in an anti-clockwise or a clockwise direction, depending upon the sign of the optical asymmetry of the system, which gives helical change in tilt direction, as described earlier for the $\mathrm{Sm} \mathrm{C}$ and $\mathrm{Sm} \mathrm{F}$ phases.
Observation of these structural patterns are supported by DSC thermograms and Phase diagram of the mixture reported by the authors [19].

\section{Conclusion}

The salient features of this investigation are the following. We have observed the occurrence of TGB-C phase in addition to the TGB-A phase, which is in accordance with Renn and Lubbensky theoretical model. In addition to this, we have observed smectic phases in some concentrations of the mixture. The higher concentrations of the given smectic liquid crystal exhibit TGB-A and TGB-C phases along with polymorphic smectic phases like Sm A, Sm I, Sm F and chiral Sm G phases.

\section{Acknowledgements}

TNG and HRS are thankful to the Department of Collegiate Education, Karnataka for permitting to carry out research work. They are also thankful to the Principal and Staff of the Government College (Autonomous), Mandya for the encouragement and support to carry out research work in the Research Centre of the institution. Assistance from Dr. J. Mahadeva and Dr. P.M. Sathyanarayana is also acknowledged.

\section{References}

[1] Doane J.W., West J.B., Whitehead J.B. and Fredly D.S. (1990) SID Digest Rep, 21, 224.

[2] Drzaic P.S., Wiley R. and Mccoy J. (1989) Proc. SPIE, 1080, 41

[3] Bahadur B. (1995) World Scientific, Singapore, Liquid Crystals-Applications and uses. Vol. I.

[4] Renn S.R. (1992) Phy. Rev. A, 45, 953.

[5] Renn S.R. and Lubbensky T.C. (1988) Phy. Rev. A, 38, 2132.

[6] de Gennes P.G. (1972) Solid State Commun, 10, 753 .

[7] de Gennes P.G. (1973) Solid State Commun, 14, 997.

[8] Abrikosov A. A. (1957) Zh ESSP Teor Fiz, 32, 1442.

[9] Goodby J.W., Waugh M.A., Stein S.M., Chin, E., Pindak R. and Patel J.S. (1989) Nature, 337, 449.

[10] Goodby J.W., Waugh M.A., Stein S.M., Chin E., Pindak R. and Patel J.S. (1989) J. Am. Chem. Soc, 111, 8119.

[11] Chenn J. and Lubbensky T.C. (1976) Phy. Rev. A, 14, 1202.

[12] Nagappa, Mahadeva J., Hanumantha Naik R. and Alapati P.R. (1997) Mol. Cryst. Liq. Cryst. 304, 409.

[13] Sadashiva B.K. (1999) Pramana-J. Phy. A, 53, No-1, 213.

[14] Pramod P.A., Prathiba R. and Madhusudan N.V. (1997) Curr. Sci. 3, 61.

[15] Ribeiro A., Barois P., Galerne Y., Oswald L. and Guillon D. (1999) Eur. Phys. J., B11, 121.

[16] Sackmann H. (1979) J. Phys. (Paris), 40, 5. 
[17] Gane P.A.C., Leadbetter A.J. and Wrighton P.G. (1981) Mol. Cryst. Liq. Cryst., 66, 247.

[18] Leadbetter A.J., Mazid M.A., Kelly, B.A., Goodby J.W. and Gray G.W. (1979) Phys. Rev. Lett., 43, 630.

[19] Govindaiah T.N., Nagappa, Sathyanarayana P.M., Mahadeva J. and Sreepad H.R. Mol. Cryst. Liq. Cryst. (In press). 